\author{
MITSUBISHI ELECTRIC RESEARCH LABORATORIES \\ http://www.merl.com
}

\title{
An MMI-Based Wavelength Combiner employing a refractive index step
}

\author{
Kojima, K.; Koike-Akino, T.; Wang, B.; Singh, S.; Ozbayat, S.; Parsons, K.; Nishikawa, S.; \\ Yagyu, E
}

TR2014-035 April 2014

\begin{abstract}
A novel wavelength combiner using a refractive index step within a multimode interference device is proposed and simulated. An InP-based 1.30/1.31 um combiner has a length of $1272 \mathrm{um}$ and an insertion loss of $0.6 \mathrm{~dB}$.

Optics Express
\end{abstract}

This work may not be copied or reproduced in whole or in part for any commercial purpose. Permission to copy in whole or in part without payment of fee is granted for nonprofit educational and research purposes provided that all such whole or partial copies include the following: a notice that such copying is by permission of Mitsubishi Electric Research Laboratories, Inc.; an acknowledgment of the authors and individual contributions to the work; and all applicable portions of the copyright notice. Copying, reproduction, or republishing for any other purpose shall require a license with payment of fee to Mitsubishi Electric Research Laboratories, Inc. All rights reserved.

Copyright (C) Mitsubishi Electric Research Laboratories, Inc., 2014

201 Broadway, Cambridge, Massachusetts 02139 



\title{
An MMI-based wavelength combiner employing a refractive index step
}

\author{
Keisuke Kojima, ${ }^{1, *}$ Toshiaki Koike-Akino, ${ }^{1}$ Bingnan Wang, ${ }^{1}$ Siddharth Singh, ${ }^{1,2}$ \\ Selman Özbayat ${ }^{1,3}$ Kieran Parsons, ${ }^{1}$ Satoshi Nishikawa, ${ }^{4}$ and Eiji Yagyu ${ }^{4}$ \\ ${ }^{I}$ Mitsubishi Electric Research Laboratories, 201 Broadway, Cambridge, MA, 02139, USA \\ ${ }^{2}$ Dept. of Electrical and Computer Eng., Stony Brook University, NY, 11794, USA \\ ${ }^{3}$ Electrical and Computer Eng. Dept., Univ. of Massachusetts Amherst, Amherst, MA, 01003 \\ ${ }^{4}$ Advanced Technology R\&D Ctr., Mitsubishi Electric Corp., 8-1-1 Tsukaguchi-Honmachi, Amagasaki, Hyogo, Japan \\ *kojima@merl.com
}

\begin{abstract}
A novel wavelength combiner using a refractive index step within a multimode interference device is proposed and simulated. An InP-based 1.30/1.31 $\mu \mathrm{m}$ combiner has a length of $1272 \mu \mathrm{m}$ and an insertion loss of $0.6 \mathrm{~dB}$.
\end{abstract}

OCIS codes: 230.1360 Beam splitter; 130.7408 Wavelength filtering devices; 230.3120 Integrated optics devices.

\section{Introduction}

Wavelength combiners are essential components for wavelength division multiplexing (WDM) optical communications systems. There have been interests in InP-based photonic integrated circuits where multiple lasers, modulators and beam combiners can be monolithically integrated on a single chip for WDM optical communications systems. It is therefore desirable to design wavelength combiners on InP substrates. Several device concepts have been utilized to realize wavelength combiners such as arrayed waveguide gratings (AWG) [1], Mach-Zehnder interferometers (MZI) [2,3] and multimode interference (MMI) devices [4], to name a few important ones.

For general power splitting or combining, MMI devices are particularly attractive as they offer several advantages such as robustness to fabrication process variations, ease of fabrication, compact size and low excess loss. Although novel MMI-based wavelength combiners have been proposed for InGaAsP/InP and silicon-on-insulator (SOI), they have typically targeted very large wavelength spacing of $240 \mathrm{~nm}$ such as $1.31 / 1.55 \mu \mathrm{m}$ where it is much easier to design shorter devices [5-7].

In this paper, we first report a scheme of using many (up to 14 pieces) relatively small patches with different refractive index within an MMI, and use a computer optimization algorithm to design efficient two-beam and fourbeam wavelength combiners. By closely examining the beam propagation patterns of the two beam combiner, it was deduced that the collective refractive index pattern acted as an interferometer. We then propose a novel and simplified MMI device concept in which non-uniform refractive index forms two distinct modes and its middle section acts as an interferometer.

\section{Devices with multiple patches}

We first designed a two-beam combiner with 14 small (length $<500 \mu \mathrm{m}$ ) patches using an optimizer. The top view is shown in Fig. 1(a). The cross-sectional view is shown in Fig. 1(b), where thinner core layer regions give lower local effective refractive index.

For the optimization, we used a metric of $\min \left(P_{1}^{\lambda_{1}}, P_{2}^{\lambda_{2}}\right)$, where $P_{1}^{\lambda_{1}}$ and $P_{2}^{\lambda_{2}}$ are the transmittances from the 1 st and 2nd input waveguide at wavelength $\lambda_{1}$ and $\lambda_{2}$, respectively. Transmittance is the ratio of the fundamental output mode power to the input fundamental mode power, calculated from the overlap integral including phase. This choice of metric means that we try to maximize the worst of the transmittance at two wavelengths. We randomly placed arbitrary sized 14 patches of low refractive index within the MMI. While fixing the MMI width to $8 \mu m$, we optimized the length of MMI, widths and positions of input and output ports, and size and location of the 14 patches. In order to optimize a total of 69 parameters simultaneously, we used 2D finite difference method for very fast simulation, combined with a covariance matrix adaptation evolutionary strategy (CMA-ES) [8]. As a result, we obtained the worst case transmittance of $83.8 \%$ (insertion loss of $0.77 \mathrm{~dB}$ ).

The propagation patterns of the optimized device are shown in Fig. 2(a) and (b). These suggest that this device can be divided into three functional sections: a $2 \times 2$ coupler, two parallel waveguides, and a $2 \times 1$ coupler. 


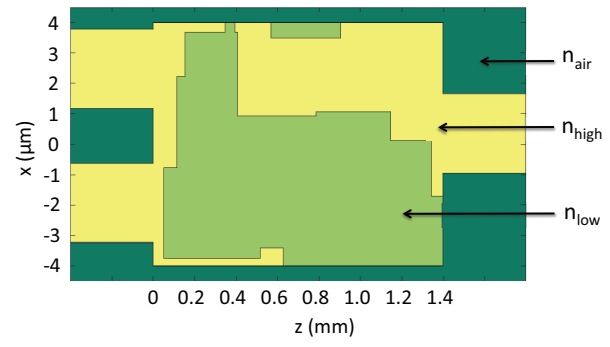

(a) Top view

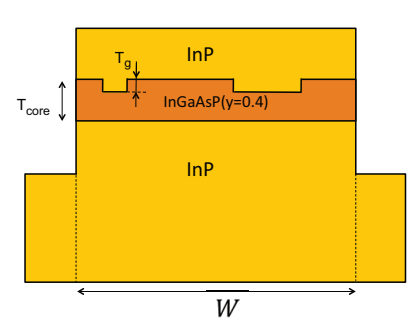

(b) Cross-sectional view

Fig. 1. Structure of the $2 \times 1$ wavelength combiner with 14 patches.

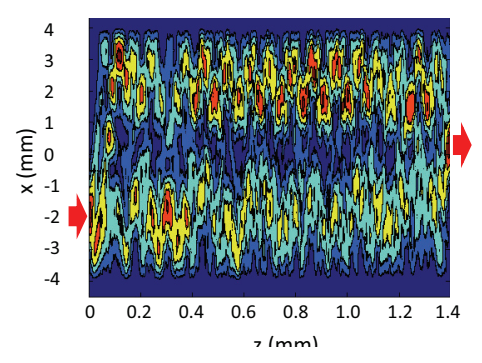

(a) $1.27 \mu \mathrm{m}$ input to port 1

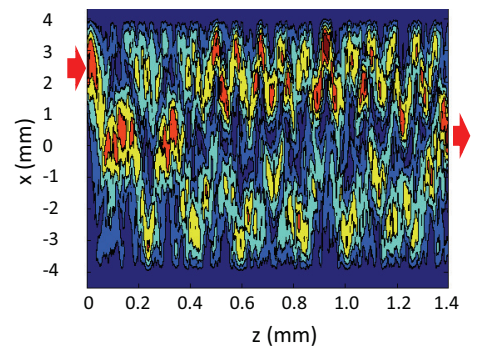

(b) $1.29 \mu \mathrm{m}$ input to port 2

Fig. 2. Propagation patterns of the input signals.

\section{Simplified two-beam combiner}

Motivated by the previous results, we propose a simplified device. The top view of the proposed device is shown in Fig. 3(a), where the lighter green part shows the lower refractive index region, and the rest constitutes the higher refractive index regions.

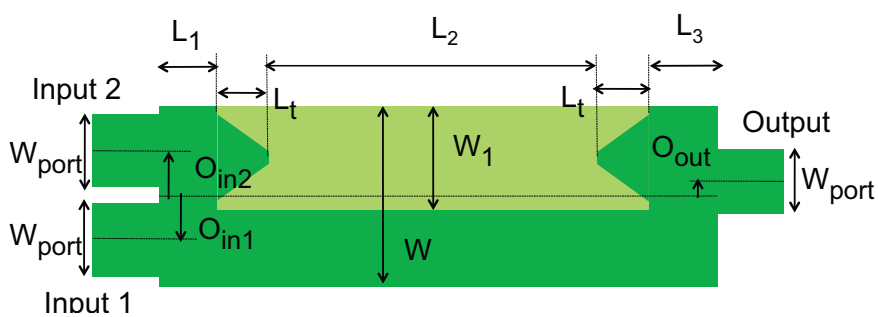

(a) Schematic view with dimensions.

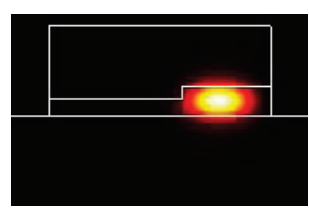

(b) The lowest TE mode

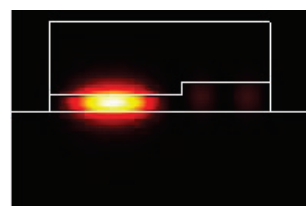

(c) The 3rd-lowest TE mode

Fig. 3. Simplified two beam combiner structure and modes at the MMI cross-section.

The cross-sectional view of the interferometer section is similar to Fig. 1(b), except that here there is a groove on one side. This creates nearly-localized propagation modes with distinct effective refractive indices in the MMI, unlike conventional uniform MMIs. Figures 3(b) and 3(c) show the lowest TE mode and the third lowest TE mode respectively, when the total MMI width is $W=6.0 \mu \mathrm{m}$, the patch width is $W_{1}=3.6 \mu \mathrm{m}$, core layer thickness is $T_{\text {core }}=$ $0.5 \mu \mathrm{m}$, and groove thickness is $T_{\mathrm{g}}=0.2 \mu \mathrm{m}$. Since the interferometer is not symmetric in terms of mode positions, instead of using symmetric $2 \times 2$ and $2 \times 1$ couplers, we use an optimization algorithm to design the asymmetric $2 \times 2$ and $2 \times 1$ couplers. With CMA-ES, we optimized widths and offsets of input/output waveguides and the lengths of the $2 \times 2$ coupler, unbalanced interferometer, $2 \times 1$ coupler and taper sections simultaneously. Considering the fabrication repeatability, the width of the tip of the taper is set to $0.2 \mu \mathrm{m}$. In the following section, we present simulation results for a 1.30/1.31 $\mu \mathrm{m}$ wavelength combiner.

The proposed device is simulated using the commercial software FIMMWAVE [9], which uses the threedimensional eigenmode expansion method to solve the propagation problem. The width of the optimized waveguides is $W_{\text {port }}=2.8 \mu \mathrm{m}$, and the total MMI length is $1271.7 \mu \mathrm{m}$.

Figures 4(a) and 4(b) show propagation patterns for $1.30 \mu \mathrm{m}$ input to port 1 , and $1.31 \mu \mathrm{m}$ input to port 2, re- 
spectively. In the interferometer section, two beams are confined into each section. However, since the two modes, as shown in Figs. 3(b) and 3(c), are not completely spatially separated, there are interference patterns as can be seen in the interferometer section. The wavelength-dependent transmission for this device is shown in Fig. 4c. Since this device was optimized at $1.30 \mu \mathrm{m}$ and $1.31 \mu \mathrm{m}$, the transmittance (ratio of the output power to the input power) is as high as 0.870 ( $0.6 \mathrm{~dB}$ loss).

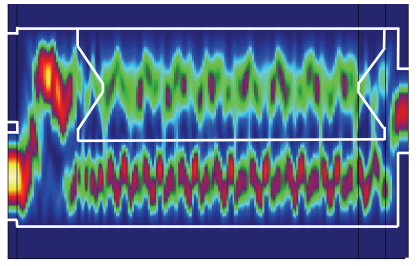

(a) $1.30 \mu \mathrm{m}$ input to port 1 .

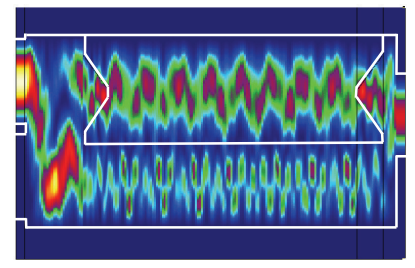

(b) $1.31 \mu \mathrm{m}$ input to port 2 .

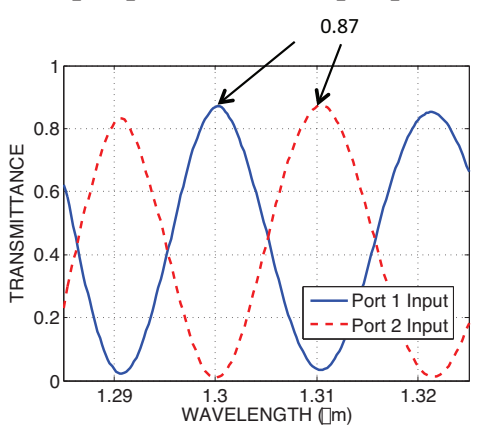

(c) Transmittance vs. wavelength.

Fig. 4. Propagation patterns and wavelength scan for a 1.30/1.31 $\mu \mathrm{m}$ wavelength combiner.

\section{Conclusion}

A novel device concept for designing compact MMI-based wavelength combiners has been proposed. A refractive index step in the cross-section of the MMI creates two distinct modes, which can be used for an unbalanced interferometer. This was enabled by the initial study of computer optimization of small patches. A wavelength combiner was designed for 1.30/1.31 $\mu \mathrm{m}$ as an example. Simulations show that the devices have an insertion loss of $0.6 \mathrm{~dB}$. This is significantly shorter than conventional MMI-based wavelength combiners. Since their width is much smaller than conventional AWGs and MZIs, they have a potential for high-density integration on a chip.

\section{References}

1. Y. Barbarin, X. J. M. Leijtens, E. A. J. M. Bente, C. M. Louzao, J. R. Kooiman, and M. K. Smit, "Extremely Small AWG Demultiplexer Fabricated on InP by Using a Douoble-Etch Process," PTL 16, 2478-2480 (2004).

2. A. Tervonen, P. Poyhonen, S. Honkanen, and M. Tahkokorpi, "A guided-wave Mach-Zehnder interferometer structure for wavelength multiplexing," PTL 3, 516-518 (1991).

3. C. van Dam, M. R. Amersfoort, G. M. ten Kate, F. P. G. M. van Ham, and M. K. Smit, "Novel InP-based phased-array wavelength demultiplexer using a generalized MMI-MZI configuration," Proc. 7th Eur. Conf on Int. Opt. (ECIO '95) 275-278 (1995).

4. L.B. Soldano and E.C.M. Pennings, "Optical multi-mode interference devices based on self-imaging: principles and applications,"JLT 13, 615-627 (1995).

5. C. Yao, H.G. Bach, R. Zhang, G. Zhou, J.H. Choi, C. Jiang, and R. Kunkel, "An ultracompact multimode interference wavelength splitter employing asymmetrical multi-section structures," Opt. Exp. 20, 18248-18253 (2012).

6. Y. Shi, S. Anand, and S. He, "A Polarization-insensitive 1310/1550-nm Demultiplexer based on Sandwiched Multimode Interference Waveguides," PTL 19, 1789-1791 (2007).

7. M-C. Wu and S-Y. Tseng, "Design and simulation of multimode interference based demultiplexers aided by computer-generated planar holograms," Opt. Exp. 18, 11270-11275 (2010).

8. M. D. Gregory, Z. Bayraktar, and D. H. Werner, "Fast Optimization of electromagnetic design problems using the covariance matrix adaptation evolutionary strategy," IEEE Trans. Antennas Propagat. 59 (4), 1275-1285 (2011).

9. D. F. G. Gallagher and T. P. Fellici, "Eigenmode expansion methods for simulation of optical propagation in photonics: pros and cons," Proc. SPIE 4987 69-82 (2003). 